\title{
THE DIAGNOSIS AND TREATMENT OF PYELITIS IN INFANCY *
}

ROWLAND G. FREEMAN, M.D.

Adjunct-Professor of Pediatrics University and Bellevue Hospital Medical School. NEW YORK

The pyelitis of infancy, which is a fairly common disease, is generally understood by pediatricians, as expressed in their writings, as a disease characterized by an active, remitting temperature, and which is treated most successfully by the use of alkalies, while some cases may be cured by hexamethylenamin in doses of $1 / 2$ to 2 grains three times a day, or every three hours.

Recent experiences have led me to believe that such statements should be materially modified. We should rather say that the pyelitis of infancy is a disease which is usually characterized by a high remitting temperature, but may give rise to no temperature, and that while some patients may be cured safely by neutralization of the urine with alkalies and others by doses of hexamethylenamin such as those named, that the most efficient treatment in difficult cases is by the use of very large doses of hexamethylenamin aided by vaccines, either commercial or autogenous. In confirmation of this statement I wish to present briefly three cases of pyelitis which I have recently had under observation.

\section{CASE REPORTS}

CASE 1.-A healthy female child, 1 year old. One month after a vaccination, which took well, and when it was practically healed the child suddenly had a temperature of $104 \mathrm{~F}$. and examination of the urine showed a pyelitis. Alkaline treatment was prescribed and in four days the urine had cleared and the child had a normal temperature. Nine days later, however, the temperature again arose and the alkaline treatment was prescribed, but after three days, the temperature having gradually risen to $105.6 \mathrm{~F}$., the alkaline treatment was stopped and hexamethylenamin was given in doses of 1 grain every four hours. (Chart 1.) This was changed two days later to 1 grain every three hours and again in two days to 1 grain every two hours, and finally to 1 grain every hour, without any evident effect on the symptoms. The hexamethylenamin was then stopped, it being considered unsafe to continue this dose for many days. Potassium citrate in doses of 5 grains every three hours was again ordered and it neutralized the urine, but under this treatment the temperature began to rise again. At this time a blood examination showed 22,000 white corpuscles, 56 per cent. polynuclears, 3,800,000 red cells and no malarial organisms. The urine drawn by a catheter showed colon bacilli as well as a few colonies of streptococci. Thirty millions of commercial colon vaccines were then administered. Administration of the vaccine was followed in two days by a normal temperature, which, however, quickly reacted up, on the fourth day having reached nearly $102 \mathrm{~F}$, so

* Submitted for publication May 15, 1913. 
that on the fifth day a second dose of $50,000,000$ colon vaccines was administered, followed immediately by a normal temperature.

The child was then put on benzoate sodium with hexamethylenamin, 12 grains of each during the day. The following day this was increased to 15 grains and the day after to 24 grains. On this day, however, the temperature again arose to $101.5 \mathrm{~F}$. On the twentieth day of the disease the child's weight was $211 / 4$ pounds, showing a gain of $21 / 4$ pounds during the preceding four weeks. The child began to gain weight as soon as the temperature was reduced by the vaccines. The following day, the temperature having risen to $101.4 \mathrm{~F} ., 40,000,000$ bacteria were again administered with a prompt reduction in the temperature. The urine continued to show many leukocytes and bacteria. Twenty-four grains of hexamethylenamin daily was continued until the twenty-seventh day of the disease when the following report on the urine was received from Dr. F. C. Wood: "The only Gram-negative bacillus which was found was not the colon, as it did not ferment sugar. There were ordinary staphylococci in small numbers. The specific gravity was 1.010. It showed no albumin or diacetic acid, but many leukocytes and bacteria."

All medication was now stopped. Six days later, the temperature having again arisen to $102.2 \mathrm{~F}$., $30,000,000$ of the same vaccines were administered. No

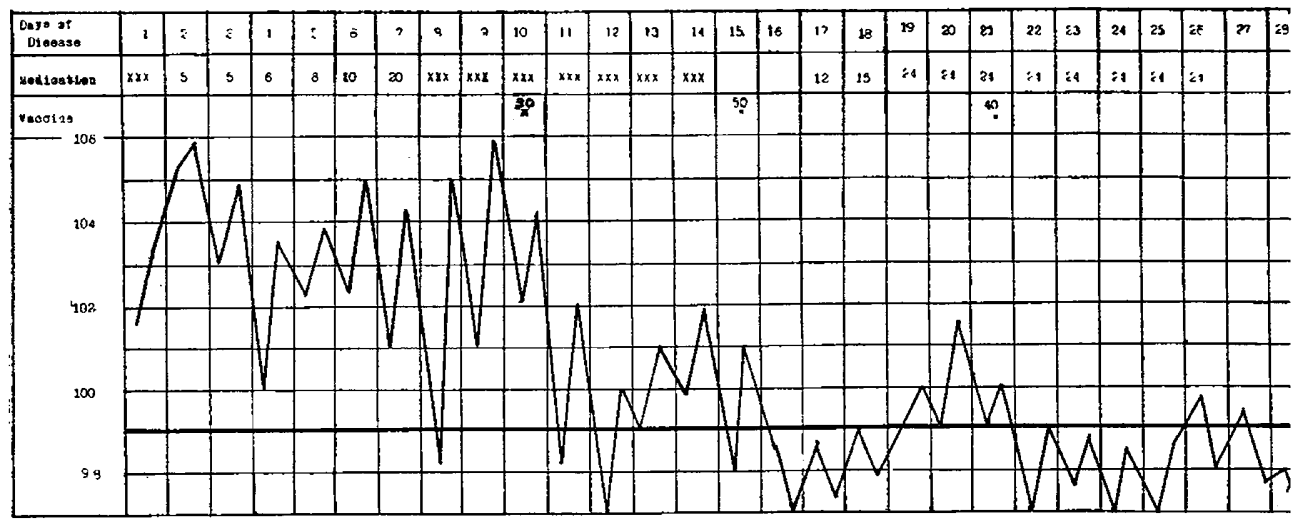

Chart 1.-(Case 1) Temperature curve in an infant with pyelitis. $\mathrm{X}=$ millions of vaccine.

further medication was attempted until the forty-second day when it was decided to try the effect of very large doses of hexamethylenamin for short periods. Twenty-five grains daily without sodium benzoate were then given for four days with a marked diminution in the number of leukocytes and bacteria present. On the fifth day this was run up to 30 grains and on the sixth day to 35 grains, with still a diminution in the bacteria and leukocytes, but without obtaining a sterile urine and without any evidence of irritation of the kidneys. There was no albumin in the urine, and no diacetic acid. On the fiftyfourth day of the disease, after a week without treatment, 40 grains of hexamethylenamin were administered with an improvement in the urinary condition, and on the following day 40 grains and the third day 45 grains, at which time the urine was found to be sterile. Treatment was then stopped and no recurrence of the urinary infection has occurred during the year that has passed.

These large doses of hexamethylenamin neither caused any evidence of disturbance of the kidneys nor any interference in the well-being of the child. As soon as the fever was controlled by the vaccines the child 
gained in weight and really seemed better when on large doses of hexamethylenamin than at any other time.

CASE 2.-It is interesting to note the long period of lack of fever and con. stitutional symptoms, but with a continuation of the pyelitis as evidenced by pus and bacteria in the urine after the administration of vaccines, especially in connection with the next case of which $I$ wish to speak in which a similar infection of the urinary tract with colon bacilli occurred in a child 9 months old, who seemed apparently well at the time the urinary infection was found. This child had been under my care since birth and had never had a febrile disturbance.

Dec. 21, 1912, I found that the urine of this apparently healthy child was moderately acid, had a specific gravity of 1.015, contained a faint trace of albumin, a moderate amount of acetone, a faint trace of phenol, clumps of pus and many bacteria, some in chains. The child was put on potassium citrate, 5 grains every three hours. A specimen taken by catheter showed a pure culture of colon bacilli.

After five days of this alkaline treatment, there being no improvement in the condition of the urine, $1 / 2$ grain of hexamethylenamin was ordered to be given every three hours, so that the child got three grains that day, on the twenty-

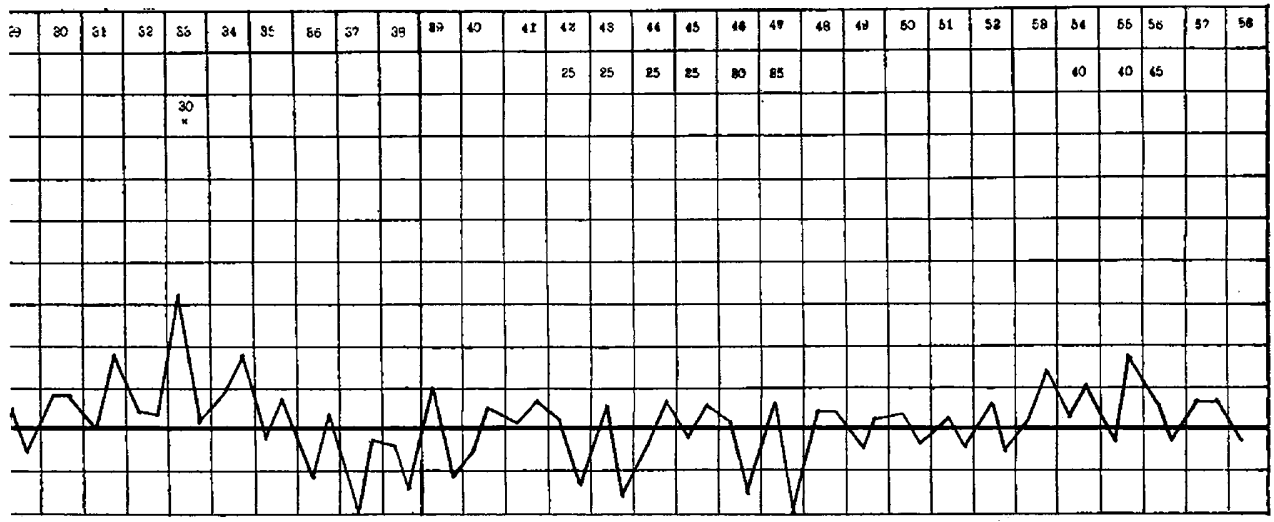

abic numerals = grains of hexamethylenamin. Roman numerals = grains of citrate of potash.

seventh 5 grains, on the twenty-eighth 10 grains, on the twenty-ninth 12 grains, on the thirtieth 15 grains, and 18 grains on the thirty-first. During this time the urine continued to contain leukocytes and bacteria, the leukocytes varying in number from 12 to 50 in a D field. On January 1, 20 grains of hexamethylenamin were administered, on the second 25 grains, and on the third 30 grains. This dose was continued until the sixth of January, when the urine still continued to be contaminated. The hexamethylenamin was stopped and potassium citrate was again administered. The contamination of the urine continued under this treatment, although several times the urine appeared almost free from contamination, and on this account the alkaline treatment was continued longer than it otherwise would have been. On January 27 four doses of 7 grain each, or 28 grains of hexamethylenamin were given, and on the twentyeighth 35 grains, on the twenty-ninth 35 grains. On the thirtieth macules appeared on the body, especially adjacent to the upper part of the diaper, and the child had a temperature of $101 \mathrm{~F}$. the night before. She was not taking her bottles very well and had lost $1 / 2$ pound in four days and the hexamethylenamin was stopped. 
With these large doses of hexamethylenamin the urine rapidly cleared and the day after it was stopped a specimen of urine was found to be sterile and no contamination of the urine has since occurred.

This case, then, of colon pyelitis without temperature in an 18-pound baby was cured by 35 grains of hexamethylenamin a day for three days without any evidence of irritation of the kidney or marked interference of the general health. Whether the macules on the body, the loss of half a pound in weight, and the slight rise in temperature were due to the hexamethylenamin I am unable to say.

No vaccines were used in this case as vaccines appear to control the constitutional symptoms and to have little or no control over the inflammation.

CASE 3.-A third case was recently seen by me in consultation with Dr. Thomas F. Lancer. A female child, 6 months old, had been sick six days. On the day preceding the one when $I$ saw the child the temperature had reached 106 (Chart 2). There was a pause at the end of inspiration and an expiratory grunt, and the movements from the bowels contained some mucus.

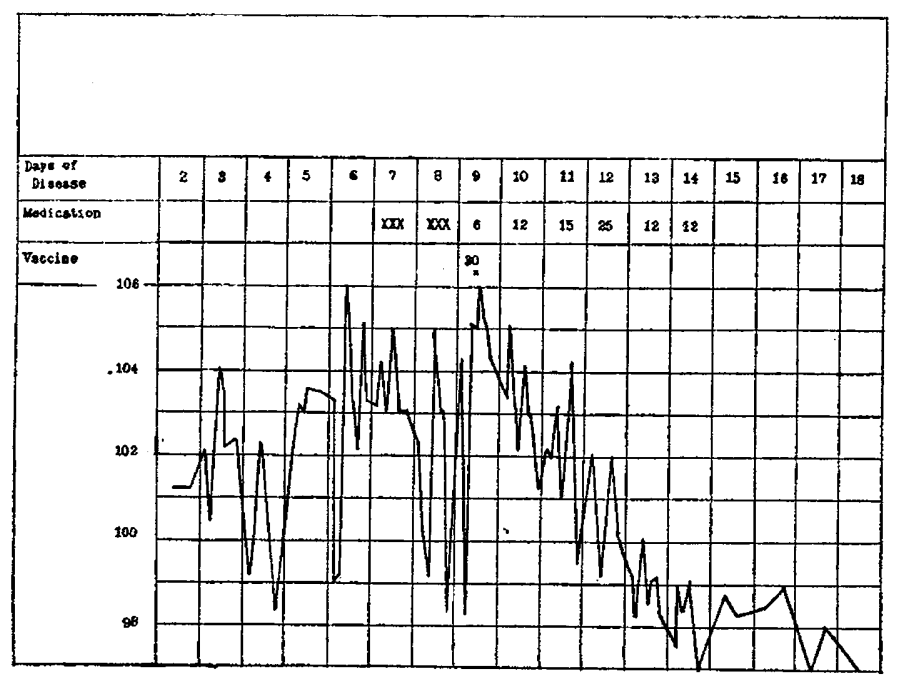

Chart 2.-(Case 3) Temperature curve in an infant with pyelitis. $\mathrm{X}=$ mil lions of vaccine. Arabic numerals = grains of hexamethylenamin. Roman numerals = grains of citrate of potash.

By a system of exclusion a probable diagnosis of pyelitis was made, and the child was put on potassium citrate. An examination of the urine showed a colon pyelitis. After three days on potassium eitrate, although the temperature had been lower, the child looked much worse and the temperature arose again to 106 F. Thirty million colon bacilli were then administered and 6 grains of hexamethylenamin were given on this day. No immediate marked decline in temperature followed the administration of vaccines. The following day 12 grains of hexamethylenamin were given, on the eleventh day 15 grains, on the twelfth day 25 grains, and on the thirteenth day the temperature was normal and the urine was sterile.

No disturbance of the child's health was caused by the large doses of hexamethylenamin, 25 grains a day in a 6-months' baby. The urine never contained more than a faint trace of albumin. 


\section{CONCLUSIONS}

Pyelitis in infancy, due to the invasion of the pelvis of the kidney with colon bacteria resulting in a purulent inflammation, can apparently occur with no perceptible rise of temperature at any time. These cases should be treated and cured by the means ordinarily used in pyelitis.

The alkaline treatment of pyelitis, while it is safe and will control many cases, is markedly less efficient than other methods of treatment.

Vaccines, either autogenous or commercial, are useful in controlling the constitutional symptoms of pyelitis.

Hexamethylenamin, while sometimes effective in doses of from $1 / 2$ to 2 grains several times a day, will not in these doses cure certain cases which may be controlled by very large doses.

Hexamethylenamin should always be administered in small doses first, but the dose should be rapidly run up, the child and its urine being carefully watched for symptoms of irritation of the kidneys.

Large doses of hexamethylenamin should not usually be continued for more than a week at a time, and then after several days without any treatment or with alkaline treatment it should be started at the maximum dose given before and the amount increased daily until an influence on the urine is obtained. Doses of 25 grains daily in a child of 6 months, and from 35 to 45 grains a day in a child from 9 to 12 months may be safely given in this way to some infants.

211 West Fifty-seventh Street. 